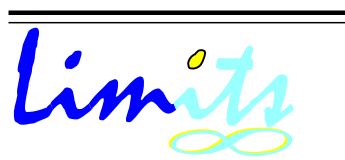

J. Math. and Its Appl.

ISSN : $1829-605 \mathrm{X}$

Vol. 1, No. 2, Nov. 2004, 9-19

\title{
On Elementary Differences Among Jump Classes
}

\author{
Yang Yue \\ Department of Mathematics, Faculty of Science \\ National University of Singapore \\ Lower Kent Ridge Road, Singapore 119260 \\ email: matyangy@nus.edu.sg
}

\section{Ringkasan}

We review recent developments in the study of jump classes in computably enumerable degrees, with special emphasis on the elementary differences among the jump classes.

Keywords: Computably enumerable degrees, jump classes

In this survey paper, we shall focus on results concerning jump classes in computably enumerable degrees. As we only consider selected topics in the area, the reader who is interested in recent developments of the subject in general may refer to [3] and [6]. The latter also gives a comprehensive list of open problems. For background material on computably enumerable sets and degrees, we recommend the classic textbook of Soare [20].

We shall roughly follow the time line, from the birth of the subject in the 1930's to the present day. Various notions and terminologies will be introduced along the way. We will end the paper with some open problems.

\section{Model of Computation and Jump Operator}

Computability Theory (also known as recursion theory) was born in the 1930's, largely due to the works of Gödel, Church and Turing. One of the motivating forces 
was to answer various decision problems. In general, a decision problem seeks an answer to the following: Given a set $A$ of natural numbers, find an algorithm which, on input $n$, decides whether $n$ is in $A$. Answering such questions naturally requires a formal definition of algorithms. In the 1930's, several definitions, arising from Gödel's notion of general recursive functions, Church's $\lambda$-calculus, and Turing's model of computation known as a Turing machine, were proposed. It turns out that these are all equivalent, in the sense that they all characterize the same class of decidable problems and capture the essence of the concept of computability.

Today with the popularization of computers, we may say that a set $A$ to be decidable (or computable) if there is a program executable on an idealized computer (i.e., with no time and memory limitation), so that given input $n$, it is able to tell us whether $n$ is in $A$ within a finite amount of time. For example, the set of prime numbers is computable.

There are uncountably many undecidable sets. A famous example is the socalled halting set $K$ using the idea of diagonalization. First of all, there is an effective list $\left\{\varphi_{e}\right\}, e=0,1, \ldots$ of all algorithmically computable functions. To have such a list, one must allow partial functions (whose domain is a proper subset of natural numbers) to appear. Let $\varphi_{e}(x) \downarrow$ mean $x$ is in the domain of $\varphi_{e}$. The halting set $K$ can be defined as $\left\{e: \varphi_{e}(e) \downarrow\right\}$. Although one can effectively list all elements in $K$, it is impossible to do so for elements not in $K$.

This brings us to the notion of computably enumerable (c.e.) sets. Those are sets that can be listed by computable functions. Noncomputable c.e. sets arise naturally in mathematics. For example, the theorems of first-order logic, the word problem for groups and the halting problem. For technical convenience, we code all expressions in first order language, groups theory etc. as natural numbers and by doing so, we may restrict our attention to sets of natural numbers.

There are sets harder or more complicated to compute than the computable ones. One can ask for a way to compare the complexities of noncomputable sets. This leads us to the notion of relative computability (reducibility) introduced by Turing [21]. Informally, a set $A$ is computable from another set $B$, if the membership of $A$ can be decided by a program executable on an idealized computer which has an extra hard disk containing all information about membership in $B$. Formally, we say that $A$ is Turing reducible to $B$, written $A \leq_{T} B$, if there is a Turing functional $\Phi$ such that $A=\Phi^{B}$. We can further define $A$ to be Turing equivalent to $B$, written $A \equiv_{T} B$, if both $A \leq_{T} B$ and $B \leq_{T} A$. Clearly, $\equiv_{T}$ is an equivalence relation. The equivalence class containing a set $A$ is called the Turing degree of $A$. We often denote the degree of a set $A$ by $\mathbf{a}=\operatorname{deg}(A) . A$ and $B$ having the same Turing degree informally means that they are of the same level of unsolvability or complexity. 
The computable sets form the least Turing degree, denoted by $\mathbf{0}$. The classical examples of undecidable problems, such as the set of theorems of first order logic and halting problem, turn out to be in the same degree, called the complete degree. We use $\mathbf{0}^{\prime}$ (read as zero jump) to denote this special degree. In fact, for a set $A$, the relativized halting problem $A^{\prime}=\left\{e: \Phi_{e}^{A}(e) \downarrow\right\}$ automatically produces a set not computable from $A$. Moreover, it naturally induces the so-called jump operator $\operatorname{deg}(A) \mapsto \operatorname{deg}\left(A^{\prime}\right)$ on Turing degrees. It is well-defined since it respects Turing reducibility, namely, $A \leq_{T} B$ implies $A^{\prime} \leq_{T} B^{\prime}$. Syntactically, $A^{\prime}$ corresponds to one additional quantifier over an $A$-computable predicate. The jump of a degree $\mathbf{a}$ is denoted by $\mathbf{a}^{\prime}$ and its $n$th iteration by $\mathbf{a}^{(n)}$. The jump operator has been one of the main focuses in computability theory. Before discussing the structure of local c.e. degrees, let us mention one recent breakthrough on the jump operator by Shore and Slaman [19]:

Theorem 1.1 (Shore and Slaman) The jump operator is definable in the Turing degree structure.

\section{Computably Enumerable Degrees and Jump classes}

We now focus our attention on the structure of c.e. degrees $\mathbb{R}=\left(\mathbf{R}, \leq_{T}\right)$, where $\mathbf{R}$ is the set of all c.e. degrees (i.e., those containing a c.e. set) and $\leq_{T}$ is the Turing reducibility relation. The degrees in this section shall refer to c.e. degree, unless otherwise indicated.

The earliest milestone was the work of Post [14] where he showed the existence of a greatest c.e. degree $\mathbf{0}^{\prime}$, and asked whether there was a c.e. degree other than $\mathbf{0}$ and $\mathbf{0}^{\prime}$. About 10 years later, Friedberg [5] and Muchnik [11] solved the problem independently by constructing two incomparable c.e. degrees.

In the study of Turing degrees, besides the jump operator, the join operator also plays an essential role. For Turing degrees $\mathbf{a}$ and $\mathbf{b}$ (not necessarily c.e.), the join of $\mathbf{a}$ and $\mathbf{b}$, denoted $\mathbf{a} \vee \mathbf{b}$, is defined to be the least upper bound of $\mathbf{a}$ and $\mathbf{b}$. For any two degrees $\mathbf{a}$ and $\mathbf{b}$, their join $\mathbf{a} \vee \mathbf{b}$ always exists, and is the degree of $A \oplus B=\{2 x \mid x \in A\} \cup\{2 x+1 \mid x \in B\}$ for some sets $A \in \mathbf{a}$ and $B \in \mathbf{b}$. Clearly, $\mathbb{R}$ is closed under the join operator. Consequently, $\mathbb{R}$ is an upper semi-lattice with least element $\mathbf{0}$ and greatest element $\mathbf{0}^{\prime}$.

The jump operator is known not to be injective. Thus we can classify the c.e. degrees a by means of the high/low hierarchy that measures the complexity of the jumps of $\mathbf{a}$. 
Definition 2.1 The jump classes (or the high/low hierarchy) are defined as follows:

$$
\begin{aligned}
& \mathbf{H}_{n}=\left\{\mathbf{a} \in \mathbb{R} \mid \mathbf{a}^{(n)}=\mathbf{0}^{(n+1)}\right\}, \\
& \mathbf{L}_{n}=\left\{\mathbf{a} \in \mathbb{R} \mid \mathbf{a}^{(n)}=\mathbf{0}^{(n)}\right\}
\end{aligned}
$$

where $n \geq 0$.

An element of $\mathbf{H}_{n}\left(\mathbf{L}_{n}\right)$ is called $h i g h_{n}$ (low $)$, and for $n=1$, we also call an element of $\mathbf{H}_{1}\left(\mathbf{L}_{1}\right)$ to be high (low).

Under this classification, the c.e. degrees can be measured quantitatively based on "how far" or "how close" they are to the computable degrees. For example, the "simplest" noncomputable degrees are the low ones, whereas the "most complicated" incomplete degrees are the high ones. It is well-known that

$$
\mathbf{L}_{1} \subset \mathbf{L}_{2} \subset \ldots
$$

and

$$
\mathbf{H}_{1} \subset \mathbf{H}_{2} \subset \ldots
$$

It should be noted that there are c.e. degrees which do not fall into any of these jump classes.

\section{Elementary Differences among Jump Classes}

The jump classes are distinct substructures of $\mathbb{R}=\left(\mathbf{R}, \leq_{T}\right)$ viewed as a partially ordered set. A natural question to ask is whether they are elementarily equivalent. Recall that two structures over the same language are called elementarily equivalent, if every sentence in the language true in one structure is also true in the other. The question can be rephrased as whether one can express the differences between jump classes by an elementary formula (in $\mathbb{R}$, such a formula would only involve the use of the partial order relation $\leq_{T}$ ).

An elementary difference between $\mathbf{H}_{1}$ and $\mathbf{H}_{n}(n>1)$ can be described in terms of what is called the plus-cupping properties.

In 1970s, Harrington [7] (see Fejer and Soare [4]) discovered a new class of c.e. degrees:

Theorem 3.1 (Harrington Plus-Cupping Theorem) There is a nonzero c.e. degree $\mathbf{a}$ such that for any c.e. degrees $\mathbf{x}, \mathbf{y}$, if $\mathbf{0}<\mathbf{x} \leq \mathbf{a} \leq \mathbf{y}$, then there is a c.e. degree $\mathbf{z}<\mathbf{y}$ such that $\mathbf{x} \vee \mathbf{z}=\mathbf{y}$.

We shall call the degree a in Theorem 3.1 a plus-cupping degree. Furthermore, Harrington also showed that 
Theorem 3.2 There is no high plus-cupping degree.

Recently, Angsheng Li [10] proved that the highness condition cannot be relaxed:

Theorem 3.3 There is a high $h_{2}$ plus-cupping degree.

Consequently we are able to distinguish the classes $\mathbf{H}_{1}$ and $\mathbf{H}_{n}(n \geq 2)$ by an elementary property:

Corollary 3.4 For each natural number $n>1$, the partially ordered structures $\mathbf{H}_{1}$ and $\mathbf{H}_{n}$ are not elementarily equivalent.

Next we consider the jump class $\mathbf{L}_{2}$. The elementary difference between $\mathbf{L}_{2}$ and $\mathbf{L}_{n}(n \geq 3)$ can be shown by nonsplitting properties, which we explain below.

We say that a degree a splits if there are degrees $\mathbf{a}_{\mathbf{0}}, \mathbf{a}_{\mathbf{1}}<\mathbf{a}$ such that $\mathbf{a}=$ $\mathbf{a}_{\mathbf{0}} \vee \mathbf{a}_{\mathbf{1}}$. This seemingly insignificant notion turned out to be one of the driving forces in the development of degree theory. Until today, we are still far from fully understanding the phenomena of splitting and nonsplitting in various degree structures.

Two important results were established by Sacks in the 1960's:

Theorem 3.5 (Sacks Splitting Theorem) Any nonzero c.e. degree a splits.

Theorem 3.6 (Sacks Density Theorem) Given any two c.e. degrees $\mathbf{b}<\mathbf{a}$, there is a c.e. degree $\mathbf{c}$ such that $\mathbf{b}<\mathbf{c}<\mathbf{a}$.

It is natural to ask if the two theorems above can be combined: Given any c.e. degrees $\mathbf{b}<\mathbf{a}$, are there c.e. degrees $\mathbf{b}<\mathbf{a}_{\mathbf{0}}, \mathbf{a}_{\mathbf{1}}<\mathbf{a}$ such that $\mathbf{a}_{\mathbf{0}} \vee \mathbf{a}_{\mathbf{1}}=\mathbf{a}$ ?

Although Robinson [15] showed that it was true for a low degree $\mathbf{b}$, the question was answered negatively in general by Lachlan [9]:

Theorem 3.7 (Lachlan Nonsplitting Theorem) There exist c.e. degrees $\mathbf{b}<$ $\mathbf{a}$ such that $\mathbf{a}$ is not splittable over $\mathbf{b}$. In other words, for any c.e. degrees $\mathbf{x}, \mathbf{y}$, if $\mathbf{b}<\mathbf{x}, \mathbf{y}<\mathbf{a}$, then $\mathbf{x} \vee \mathbf{y} \neq \mathbf{a}$.

It is worth mentioning that Lachlan's result had more important impact than merely settling a fundamental question. In the proof of Theorem 3.7, Lachlan introduced an extremely powerful method, now categorized as $\mathbf{0}^{\prime \prime \prime}$-priority method.

We shall refer to the pair of c.e. degrees $\mathbf{b}<\mathbf{a}$ in Theorem 3.7 as a Lachlan nonsplitting pair. 
We now return to low $_{2}$ degrees. Harrington (unpublished) and independently Bickford and Mills [1] (see Shore and Slaman [17]) showed that Lachlan nonsplitting pair does not exist in $\mathbf{L}_{2}$ :

Theorem 3.8 Every low 2 c.e. degree bounds no Lachlan nonsplitting pair.

Shore and Slaman (see Shore and Slaman [18]) have observed that the existing technical resources could show that the low $_{2}$ was the best possible:

Theorem 3.9 There exists a Lachlan nonsplitting pair $\mathbf{a}<\mathbf{1}$ such that $\mathbf{1}$ is low low $_{3}$

By combining Theorems 3.8 and 3.9, we are able to distinguish between the classes $\mathbf{L}_{2}$ and $\mathbf{L}_{n}(n \geq 3)$ by some elementary property:

Corollary 3.10 For each natural number $n>2$, the partially ordered structures $\mathbf{L}_{2}$ and $\mathbf{L}_{n}$ are not elementarily equivalent.

Before we discuss the elementary equivalence issue for other jump classes, let us take a detour to the topic on definable subsets of $\mathbb{R}$. As we shall see, the two topics are closely related. In fact, it is the study of definable ideals that leads to the Join Theorem by Jockusch, Li and Yang, which is the key to distinguishing the jump class $\mathbf{L}_{1}$ and $\mathbf{L}_{n}(n \geq 2)$.

\section{Definable Subsets in $\mathbb{R}$}

One of the central problems about $\mathbb{R}$ is to characterize the definable sets and relations of $\mathbb{R}$. A set of degrees is called definable in $\mathbb{R}$ if there is a formula, involving only the predicate $\leq_{T}$, which describes the set. A long standing open problem in computability theory is whether the structure $\mathbb{R}$ is rigid, that is, whether there is a nontrivial automorphism of $\mathbb{R}$. The study of definability is closely linked to the rigidity of $\mathbb{R}$, since any two degrees in $\mathcal{R}$ paired by an automorphism must satisfy the same definable properties, so that the existence of a particular definable relation may rule out certain automorphisms of $\mathbb{R}$.

One of the most significant results in the past decade is the definability of most of the jump classes. Using first order representation of standard models of arithmetic within $\mathbb{R}$, Nies, Shore and Slaman [12] have shown:

Theorem 4.1 For every $n>0, \mathbf{H}_{n}$ and $\mathbf{L}_{n+1}$ are definable in $\mathbb{R}$. 
The glaring exception is the jump class $\mathbf{L}_{1}$. It remains open this class is definable.

Other interesting problems in definability concern definable ideals. We say that a set of degrees $I$ is an ideal of $\mathcal{R}$ if $I$ is closed under join and $I$ is closed downwards.

Perhaps the earliest known example of a definable ideal is the collection of all noncuppable degrees. We say that a c.e. degree $\mathbf{a}$ is noncuppable if for any c.e. degree $\mathbf{x}, \mathbf{a} \vee \mathbf{x}=\mathbf{0}^{\prime}$ if and only if $\mathbf{x}=\mathbf{0}^{\prime}$. Let $\mathbf{N C u p}$ denote the set of all noncuppable degrees. It is easy to verify that $\mathbf{N C u p}$ is an ideal. Cooper and Yates in 1970's showed that NCup is nontrivial:

Theorem 4.2 (Cooper and Yates) There exists a noncuppable c.e. degree $\mathbf{a} \neq \mathbf{0}$

Although there are a few other known definable ideals, one would like to a find systematic way of generating infinitely many definable ideals. A natural attempt is to make use of the definability of jump classes and produce a sequence of new definable ideals. We offer two such attempts below. As we shall see, both attempts fail.

Attempt one is to make use of the classes $\mathbf{H}_{n}$, and to generalize the notion of noncuppable degrees as follows.

Let $\mathbf{N C u p H}=\left\{\mathbf{a}: \forall \mathbf{w}\left(\mathbf{a} \vee \mathbf{w} \in \mathbf{H}_{1} \Rightarrow \mathbf{w} \in \mathbf{H}_{1}\right\}\right.$.

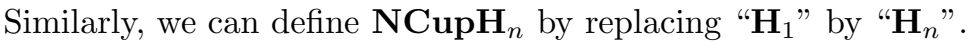

It follows from Theorem 4.1 that for each positive integer $n, \mathbf{N C u p H}_{n}$ is a definable ideal.

The second attempt is to make use of the classes $\mathbf{L}_{n}$. The starting point is the notion of almost deep degrees, introduced by Cholak, Groszek and Slaman [2]: A degree $\mathbf{a}$ is called an almost deep degree if

$$
\forall \mathbf{l} \in \mathbf{L}_{1}\left[(\mathbf{a} \vee \mathbf{l}) \in \mathbf{L}_{1}\right]
$$

In other words, joining with a preserves lowness.

They showed:

Theorem 4.3 There is a nonzero almost deep degree.

Notice that the almost deep degrees form an ideal. It is not known if it is definable as it is hinged to the definability of $\mathbf{L}_{1}$. However, one could modify the notion to regain definability as follows. 
Let $n$ be a positive integer. A degree $\mathbf{a}$ is called an almost $n$-deep degree if

$$
\forall \mathbf{l} \in \mathbf{L}_{n}\left[(\mathbf{a} \vee \mathbf{l}) \in \mathbf{L}_{n}\right] .
$$

In other words, joining with a preserves low $_{n}$-ness.

Notice that for $n>1$, by Theorem 4.1 again, the almost $n$-deep degrees do form definable ideals. It was once believed that the method of proving Theorem 4.3 could be modified to produce a nonzero almost 2-deep degree.

However, the attempts of using $\mathbf{N C u p H}_{n}$ and almost $n$-deep degrees do not work, as Jockusch, Li and Yang [8] have shown the following version of Join Theorem:

\section{Theorem 4.4 (Jockusch, A. Li and Y. Yang)}

$$
(\forall \mathbf{a} \neq \mathbf{0})(\exists \mathbf{b})\left[\mathbf{b}^{\prime \prime}=(\mathbf{a} \vee \mathbf{b})^{\prime}=\mathbf{0}^{\prime \prime}\right] .
$$

Equivalently, $(\forall \mathbf{a} \neq \mathbf{0})\left(\exists \mathbf{b} \in \mathbf{L}_{2}\right)\left[(\mathbf{a} \vee \mathbf{b}) \in \mathbf{H}_{1}\right]$.

\section{Corollary 4.5}

(i) For each $n \geq 1$, the ideal $\mathbf{N C u p H}_{n}$ is trivial, i.e., the zero ideal.

(ii) For each $n \geq 2$, the ideal of almost $n$-deep degrees is trivial.

Theorem 4.4 has other interesting consequences. One motivation came from the study of the join operator in c.e. degrees, in the spirit of Posner and Robinson [13].

Theorem 4.6 (Posner and Robinson) In Turing degrees, for any $\mathbf{0} \neq \mathbf{a} \leq \mathbf{0}^{\prime}$, there exists $\mathbf{b}$ such that

$$
\mathbf{b}^{\prime}=\mathbf{a} \vee \mathbf{b}=\mathbf{0}^{\prime} .
$$

However, by the existence of noncuppable degrees, we have no such join theorem in $\mathcal{R}$. Thus, Theorem 4.4 is the optimal result one can hope for in $\mathcal{R}$.

We are now ready to return to the topic of elementary differences between $\mathbf{L}_{1}$ and $\mathbf{L}_{n}(n>1)$. Using the fact that there exists a nonzero almost deep degree and that there is no nonzero almost $n$-deep degree (for $n>1$ ), we have the following corollary:

Corollary 4.7 For each natural number $n>1$, the partially ordered structures $\mathbf{L}_{1}$ and $\mathbf{L}_{n}$ are not elementarily equivalent.

Finally, in 2004, Richard Shore [16] used the coding technique of [12] to settle the elementary equivalence problem for all jump classes $\mathbf{L}_{n}$ :

Theorem 4.8 (Shore) For each natural number $n>m>1$, the partially ordered structures $\mathbf{L}_{n}$ and $\mathbf{L}_{m}$ are not elementarily equivalent. 


\section{Open Problems}

We end the survey paper by listing some open problems related to definability in $\mathbb{R}$.

Open Question 1 (Slaman) Is there any nontrivial definable principal ideal in $\mathbb{R}$ ?

Open Question 2 Are there infinitely many definable ideals in $\mathcal{R}$ ?.

The problem on elementary equivalence among the jump classes $\mathbf{H}_{n}$ remains open:

Open Question 3 Are there $m \neq n$ such that $\mathbf{H}_{m}$ and $\mathbf{H}_{n}$ are elementarily equivalent?

Until now, we have very limited knowledge of $\mathbf{H}_{n}$ and $\mathbf{L}_{n+1}$ for $n \geq 3$. This motivates our final open problem below. The words "naturally definable" emphasize on the simplicity of the definition, in particular, rule out the coding technique used in [12].

Open Question 4 (i) Is there a naturally definable set $A \subset \mathbb{R}$ such that $A \cap$ $\mathbf{H}_{n}=\emptyset$ but $A \cap \mathbf{H}_{n+1} \neq \emptyset$ ? We assume that $n \geq 2$. (For $n=1$, the answer is yes by existing results.)

(ii) Is there a naturally definable set $A \subset \mathbb{R}$ such that $A \cap \mathbf{L}_{n}=\emptyset$ but $A \cap \mathbf{L}_{n+1} \neq$ $\emptyset$ ? We assume that $n \geq 3$. (For $n=1,2$, the answer is yes by existing results.)

(iii) Is there a naturally definable nonempty set $A \subset \mathbb{R}$ such that $A \cap \mathbf{L}_{n}=\emptyset$ and $A \cap \mathbf{H}_{n}=\emptyset$ for all $n \geq 1$ ?

\section{Pustaka}

[1] M. Bickford and C. F. Mills. Lowness properties of r.e. degrees. Unpublished preprint, 1982.

[2] Peter Cholak, Marcia Groszek, and Theodore A. Slaman. An almost deep degree. J. Symbolic Logic, 66(2):881-901, 2001. 
[3] Peter A. Cholak, Steffen Lempp, Manuel Lerman, and Richard A. Shore, editors. Computability Theory and Its Applications: Current Trends and Open Problems, volume 257 of Contemporary Mathematics, Providence, RI, 2000. American Mathematical Society.

[4] Peter A. Fejer and Robert I. Soare. The plus-cupping theorem for the recursively enumerable degrees. In Logic Year 1979-80: University of Connecticut, pages 49-62, 1981.

[5] R. M. Friedberg. Two recursively enumerable sets of incomparable degrees of unsolvability (solution to post's problem, 1944). Proc. Nat. Acad. Sci. U.S.A., 43:236-238, 1957.

[6] Edward R. Griffor, editor. Handbook of Computability Theory, volume 140 of Studies in Logic and the Foundations of Mathematics. North-Holland Publishing Co., Amsterdam, 1999.

[7] Leo A. Harrington. Plus-cupping in the recursively enumerable degrees. notes, 1978.

[8] Carl G. Jockusch, Jr., Angsheng Li, and Yue Yang. A join theorem for the computably enumerable degrees. Trans. Amer. Math. Soc., 356:2557-2568, 2004 .

[9] Alistair H. Lachlan. A recursively enumerable degree which will not split over all lesser ones. Ann. Math. Logic, 9:307-365, 1975.

[10] Angsheng Li. Elementary differences among jump hierarchies. to appear.

[11] A. A. Muchnik. On the unsolvability of the problem of reducibility in the theory of algorithms. Dokl. Akad. Nauk SSSR, N. S. 108:194-197, 1956.

[12] André Nies, Richard A. Shore, and Theodore A. Slaman. Interpretability and definability in the recursively enumerable degrees. Proc. London Math. Soc. (3), 77(2):241-291, 1998.

[13] David B. Posner and Robert W. Robinson. Degrees joining to 0'. J. Symbolic Logic, 46(4):714-722, 1981.

[14] Emil L. Post. Recursively enumerable sets of positive integers and their decision problems. Bull. Amer. Math. Soc., 50:284-316, 1944.

[15] R. W. Robinson. Interpolation and embedding in the recursively enumerable degrees. Ann. of Math., 93:285-314, 1971. 
[16] Richard A. Shore. The $\mathrm{Low}_{n}$ and $\mathrm{Low}_{m}$ r.e. degrees are not elementarily equivalent. to appear.

[17] Richard A. Shore and Theodore A. Slaman. Working below a low 2 recursively enumerably degree. Arch. Math. Logic, 29(3):201-211, 1990.

[18] Richard A. Shore and Theodore A. Slaman. Working below a high recursively enumerable degree. J. Symbolic Logic, 58(3):824-859, 1993.

[19] Richard A. Shore and Theodore A. Slaman. Defining the Turing jump. Math. Res. Lett., 6(5-6):711-722, 1999.

[20] Robert I. Soare. Recursively Enumerable Sets and Degrees. Springer-Verlag, Heidelberg, 1987.

[21] Alan M. Turing. On computable numbers with an application to the Entscheidungsproblem. Proc. London Math. Soc. (3), 42:230-265, 1936. A correction, $43: 544-546$. 